\title{
Implementación de 9 indicadores de calidad en un laboratorio hospitalario
}

\author{
ANA MARÍA GUZMÁN D. ${ }^{1}$, TOMÁS SÁNCHEZ P., ${ }^{2, a}$, \\ RICARDO DE LA BARRA D. ${ }^{2, a}$, ANGÉLICA MADRID Q., ${ }^{2, a}$ TERESA QUIROGA G. ${ }^{1}$
}

\section{Implementation of nine quality indicators in a hospital emergency clinical laboratory}

Background: Quality indicators are tools used to monitor specific activities within a process and improve it. In the area of clinical laboratories, the National Accreditation Standards for Providers of Health and the ISO 15189 standard recommend the implementation of indicators that monitor the test cycle with emphasis on those that contribute to a safer health care. Aim: To describe the implementation of nine indicators in a hospital clinical laboratory and their measurement during one year. Material and Methods: The indicators implemented and measured were four of the pre-analytical phase (number of rejected samples, times of transport, blood culture contamination and blood cultures inoculated with adequate blood volumes), two of the analytical phase (coherence of Gram stains of blood culture with microorganism cultured and correct results in external quality control surveys) and three of the postanalytical phase (compliance with order to report lapse goals, corrected reports and alert values report). Results: Two indicators of pre-analytical phase did not meet the per determined targets: number of rejected samples and blood cultures inoculated with adequate blood volume. All indicators of the analytical and post analytical phases were within the pre-determined targets. Conclusions: Coordinated work should be initiated especially with the nursing service to correct the two indicators that did not meet the target. The incorporation of quality indicators to monitor critical processes within the laboratory was undoubtedly an opportunity to identify areas for improvement.

(Rev Med Chile 2011; 139: 205-214).

Key words: Hospital information systems; Laboratories, hospital; Quality indicators, healthcare.

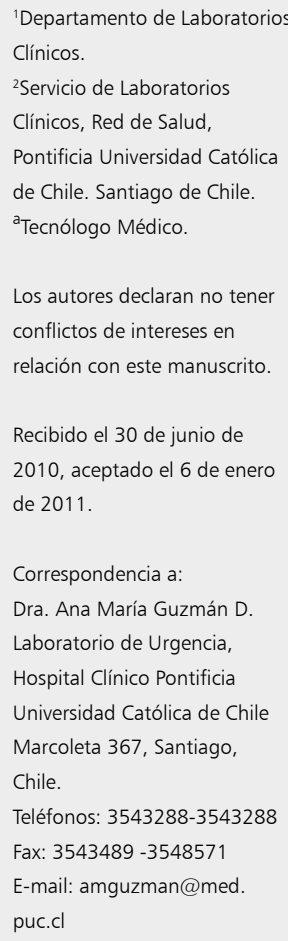

E n Medicina, así como en otras disciplinas, calidad se asocia a excelencia en el conocimiento, perfección de un proceso $\mathrm{u}$ obtención de buenos resultados. En actividades que involucran la prestación de un servicio a las personas, calidad se entiende como "hacer bien las cosas", teniendo implícitos conceptos como excelencia técnica, decisiones adecuadas y oportunas, fluidez en las comunicaciones con el paciente y su familia, eficiencia, seguridad, etc ${ }^{1}$. Según define el Instituto de Medicina de la Academia de Ciencias de los Estados Unidos de Norteamérica, calidad en salud es "el grado en que los prestadores de salud aumentan la probabilidad de obtener resultados deseados yéstos son consistentes con el conocimiento de la medicina actual"'.

El Ministerio de Salud de Chile, como una forma de asegurar la calidad de atención, ha desarrollado los Estándares de Acreditación Nacional para Prestadores de Atención Abierta (ambulatoria) ${ }^{3}$ y Cerrada (hospitalaria) ${ }^{4}$, incluyendo todos los ámbitos de la atención de salud con características obligatorias o mínimas a cumplir, para que las instituciones de salud puedan ser acreditadas. 
Estos estándares incluyen los laboratorios clínicos, donde se estipula evaluar en forma periódica las etapas pre-analítica, analítica y post-analítica.

Por otra parte, la Norma ISO (International Standard Organization) 15189, equivalente a la Norma Chilena 2547 del Instituto Nacional de Normalización "Laboratorios Clínicos- Requisitos particulares para la Calidad y Competencia" en el punto normativo 4.12.4 referido al mejoramiento continuo, exige la implementación de indicadores de calidad para evaluar y monitorizar la contribución del laboratorio a la seguridad del paciente. El Servicio de Laboratorios Clínicos de la Pontificia Universidad Católica de Chile (SLC-PUC) está acreditado bajo esta norma desde el año 2008 y tiene en funcionamiento un Sistema de Gestión de Calidad desde el año 2004.

Durante el año 2008 nuestro servicio inició la medición de una serie de indicadores de calidad con el objetivo no sólo de satisfacer los requerimientos normativos antes mencionados, sino que también identificar problemas y posibles mejoras, evaluar eficacia y logro de metas establecidas, realizar comparaciones internas y con otras instituciones, $y$ finalmente colaborar efectivamente al cuidado del paciente con información relevante para los servicios clínicos. Durante ese año se realizaron ajustes referidos a periodicidad, sistematización de la obtención de los datos, etc. para entrar en funcionamiento definitivo el año 2009.

El objetivo de este trabajo es mostrar los indicadores elaborados por el SLC-PUC relevantes para el área de atención cerrada y dar a conocer los valores obtenidos de las mediciones realizadas durante el año 2009, los cuales serán importantes para identificar áreas de mejora.

\section{Materiales y Método}

\section{Escenario}

El Laboratorio de Urgencia del Hospital Clínico UC, perteneciente al SLC-PUC realiza alrededor de 40.000 exámenes mensuales, atendiendo $90 \%$ de las necesidades totales de exámenes de pacientes hospitalizados. El hospital cuenta con 398 camas pertenecientes a 14 servicios clínicos incluyendo: Anestesia y Recuperación, Cirugía General, Cirugía Cardiovascular, Intermedio Médico Neurológico, Medicina Interna, Obstetricia y Ginecología, Unidad Coronaria, Unidad de Pacientes Críticos
(UPC) Adultos, Pediatría, Neonatología, UPC Pediátrica, Urgencia Adultos, Urgencia Pediátrica y Pensionado.

\section{Elaboración de indicadores y cálculo}

Se realizó revisión de la literatura y normativas disponibles, seleccionando en un comité directivo, los indicadores más relevantes ${ }^{6-10}$, estableciendo posteriormente un procedimiento documentado.

Para cada indicador se trabajó con una matriz o plantilla tipo que incluyó: nombre del indicador, fórmula, meta de cumplimiento, justificación teórica de su implementación, fuentes de información, metodología para su obtención, periodicidad de medición y responsables. Posteriormente, se recopiló toda la información para el cálculo de cada indicador y se analizó el desempeño de cada uno de ellos durante el año 2009, comparando los resultados con las metas establecidas.

Para efectos de esta publicación, se tomaron en consideración aquellos indicadores relevantes para la atención cerrada, obtenidos de los datos del Laboratorio de Urgencia del Hospital Clínico. En algunos casos, en los que se consideró importante, los datos fueron desglosados según servicio clínico.

\section{Resultados}

\section{Procedimiento de indicadores de calidad}

Según lo definido por el Sistema de Gestión de Calidad del SLC-PUC, el documento de Indicadores de Calidad incluyó objetivos, alcances, definiciones, referencias, responsabilidades, metodología, registros asociados, anexos y revisiones o modificaciones. Se incorporó la matriz o plantilla tipo ya mencionada y la responsabilidad del análisis de datos fue asignada al médico jefe del laboratorio. En el caso de no cumplir la meta, se definió realizar análisis causa raíz e implementar las acciones correctivas requeridas. Se especificó también, que los datos debían ser revisados por la dirección del SLC-PUC integrando los resultados de las distintas unidades y/o laboratorios para implementar acciones de mejora adicionales si se estimaba necesario.

\section{Indicadores elaborados}

Se generaron 9 indicadores, 4 de la fase preanalítica, 2 de la analítica y 3 de la post-analítica. La Tabla 1 resume la racionalidad de la implemen- 
Indicadores de calidad de laboratorio hospitalario - A. M. Guzmán et al

tación. La Tabla 2 muestra el nombre del indicador, fórmula correspondiente, meta de cumplimiento, periodicidad de las mediciones y algunas observaciones que se consideraron pertinentes.

\section{Resultados de las mediciones}

\section{Fase Pre-analitica}

La Tabla 3 muestra los resultados de los indicadores: solicitud de nueva muestra, tiempos de traslado y botellas de hemocultivos inoculadas con volumen adecuado de sangre; observándose que el primero y el tercero no cumplen con la meta establecida. Este último se encuentra muy por debajo de la meta establecida ( $80 \%$ ) con sólo $37 \%$ de cumplimiento. La Figura 1 muestra el porcentaje de contaminación de los hemocultivos, siempre por debajo del $2 \%$, cumpliendo con la meta.

Desglosado por servicio clínico de origen, el porcentaje de solicitud de nueva muestra es mayor a lo estipulado según la meta establecida $(\leq 0,5 \%)$ en todos ellos, alcanzando cifras del 2,5\%, excepto en el Servicio de Urgencia que cumple en los tres cuatrimestres y Anestesia y Recuperación que cumple en el primer y tercer cuatrimestre (Tabla 4). El porcentaje de botellas de hemocultivo inoculadas con volumen escaso y excesivo desglosado por servicio se muestra en la Tabla 5, donde se observa que los servicios de adultos inoculan sus botellas principalmente con volumen excesivo y los servicios pediátricos las inoculan con volumen insuficiente o escaso.

Tabla 1. Indicadores colocados en funcionamiento en el Laboratorio de Urgencia del hospital Clínico UC (área de atención cerrada) y su justificación teórica

\begin{tabular}{|c|c|c|}
\hline & Indicador & Justificación \\
\hline \multirow{4}{*}{ 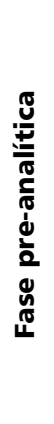 } & Solicitud de nueva muestra & $\begin{array}{l}\text { Existencia de criterios de aceptabilidad y rechazo contribuyen a la calidad } \\
\text { de los resultados generados por los laboratorios clínicos }\end{array}$ \\
\hline & Cumplimiento tiempos de traslado & $\begin{array}{l}\text { Tiempo de traslado es determinante para mantención de las condiciones } \\
\text { para análisis de muestras clínicas }\end{array}$ \\
\hline & $\begin{array}{l}\text { \% de contaminación de los hemoculti- } \\
\text { vos de sangre periférica (SP) }\end{array}$ & $\begin{array}{l}\text { Hemocultivos contaminados traducen mala técnica de antisepsia de la } \\
\text { piel, probable retraso de diagnóstico infección del torrente sanguíneo y } \\
\text { gastos innecesarios en procesamiento de botellas contaminadas }\end{array}$ \\
\hline & $\begin{array}{l}\% \text { de botellas de hemocultivo con } \\
\text { volumen adecuado de sangre }\end{array}$ & $\begin{array}{l}\text { Rendimiento del hemocultivo depende, entre otros factores, del volumen } \\
\text { de sangre inoculado en la botella }\end{array}$ \\
\hline \multirow{2}{*}{ 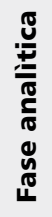 } & $\begin{array}{l}\text { \% de concordancia del gram del he- } \\
\text { mocultivo con la identificación final } \\
\text { en el cultivo }\end{array}$ & $\begin{array}{l}\text { La discordancia genera disconformidad del médico tratante, inicio de } \\
\text { un tratamiento inadecuado y posiblemente prolongación de estadía } \\
\text { hospitalaria del paciente }\end{array}$ \\
\hline & $\begin{array}{l}\% \text { de participaciones correctas en en- } \\
\text { cuestas de control de calidad externo }\end{array}$ & $\begin{array}{l}\text { Resultados correctos en las Encuestas de Calidad Externas aseguran la } \\
\text { veracidad de los resultados generados en el Laboratorio }\end{array}$ \\
\hline \multirow{3}{*}{ 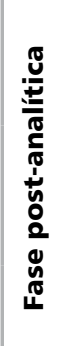 } & $\begin{array}{l}\text { \% cumplimiento de plazos de entrega } \\
(\mathrm{PE}) \text { de resultados de exámenes }\end{array}$ & $\begin{array}{l}\text { Cumplimiento en plazo de entrega responde a calidad percibida por el } \\
\text { paciente o médico. La oportunidad del resultado podría además tener } \\
\text { implicancia en el cuidado del enfermo }\end{array}$ \\
\hline & $\%$ informes corregidos & $\begin{array}{l}\text { Un examen corregido, es resultado normalmente de un reclamo o discon- } \\
\text { formidad del médico respecto al resultado erróneo, el cuál puede tener } \\
\text { repercusión en el cuidado del enfermo }\end{array}$ \\
\hline & $\begin{array}{l}\text { \% de aviso de valores de alerta (VA) al } \\
\text { médico tratante antes de } 30 \text { min }\end{array}$ & $\begin{array}{l}\text { Examen muy alterado con riesgo vital para el paciente. La comunicación } \\
\text { oportuna permite tomar una decisión médica }\end{array}$ \\
\hline
\end{tabular}


Tabla 2. Indicadores colocados en funcionamiento en el Laboratorio de Urgencia del hospital Clínico UC (área de atención cerrada), fórmula, meta de cumplimiento y observaciones de las mediciones

\begin{tabular}{|c|c|c|c|}
\hline Indicador & Fórmula & Meta & $\begin{array}{l}\text { Periodicidad y observaciones de } \\
\text { la medición }\end{array}$ \\
\hline $\begin{array}{l}\text { Solicitud de nueva mues- } \\
\text { tra }\end{array}$ & $\begin{array}{l}\mathrm{n}^{\circ} \text { de solicitudes de nueva muestra } \\
\text { X100 } \\
n^{\circ} \text { total exámenes recibidos }\end{array}$ & $\leq 0,5 \%$ & $\begin{array}{l}\text { Suma de datos acumulados por cua- } \\
\text { trimestre }\end{array}$ \\
\hline $\begin{array}{l}\text { Cumplimiento tiempos de } \\
\text { traslado }\end{array}$ & $\begin{array}{l}\mathrm{n}^{\circ} \text { exámenes recibidos en tiempo ade- } \\
\text { cuado } \times 100 \\
n^{\circ} \text { total de exámenes recibidos }\end{array}$ & $\geq 80 \%$ & Datos meses abril, agosto y diciembre. \\
\hline $\begin{array}{l}\% \text { de contaminación de } \\
\text { los hemocultivos de san- } \\
\text { gre periférica (SP) }\end{array}$ & $\begin{array}{l}\mathrm{n}^{\circ} \text { botellas hemocultivo contaminadas } \\
(\mathrm{SP}) \mathrm{x} 100 \\
\mathrm{n}^{\circ} \text { total de botellas de hemocultivo } \\
\text { (SP) tomadas }\end{array}$ & $\leq 2 \%$ & Datos acumulados por mes \\
\hline $\begin{array}{l}\% \text { de botellas de hemo- } \\
\text { cultivo de sangre periféri- } \\
\text { ca con volumen adecuado }\end{array}$ & $\begin{array}{l}\mathrm{n}^{\circ} \text { botellas hemocultivo (SP) con volu- } \\
\text { men adecuado } \times 100 \\
\mathrm{n}^{\circ} \text { total botellas hemocultivo tomadas }\end{array}$ & $\geq 80 \%$ & $\begin{array}{l}\text { Suma de datos acumulados por cua- } \\
\text { trimestre }\end{array}$ \\
\hline $\begin{array}{l}\text { \% de concordancia del } \\
\text { gram del hemocultivo con } \\
\text { la identificación final en el } \\
\text { cultivo. }\end{array}$ & $\begin{array}{l}\mathrm{n}^{\circ} \text { Gram hemocultivo concordantes } \\
\text { con cultivo } \times 100 \\
\mathrm{n}^{\circ} \text { total Gram de hemocultivos rea- } \\
\text { lizados }\end{array}$ & $\geq 98 \%$ & $\begin{array}{l}\text { Suma de datos acumulados por se- } \\
\text { mestre }\end{array}$ \\
\hline $\begin{array}{l}\% \text { de participaciones co- } \\
\text { rrectas en encuestas de } \\
\text { control de calidad externo }\end{array}$ & $\begin{array}{l}\mathrm{n}^{\circ} \text { determinaciones aceptadas en en- } \\
\text { cuestas externas } \times 100 \\
\mathrm{n}^{\circ} \text { total determinaciones realizadas en } \\
\text { encuestas externas }\end{array}$ & $\geq 95 \%$ & $\begin{array}{l}\text { Suma de datos acumulados del año. } \\
\text { Encuestas de calidad CAP* y PEEC, } \\
\text { ISP** }\end{array}$ \\
\hline $\begin{array}{l}\text { \% cumplimiento de plazos } \\
\text { de entrega }(\mathrm{PE}) \text { de resulta- } \\
\text { dos de exámenes }\end{array}$ & $\begin{array}{l}\mathrm{n}^{\circ} \text { exámenes informados dentro de } \\
\text { plazo entrega } \times 100 \\
\mathrm{n}^{\circ} \text { exámenes recibidos }\end{array}$ & $\geq 80 \%$ & $\begin{array}{l}\text { Datos meses abril, agosto y diciembre } \\
\text { para la totalidad de los exámenes } \\
\text { provenientes del Servicio de Urgencia. } \\
\text { Plazos de entrega definidos por Subdi- } \\
\text { rección Técnica del Servicio }\end{array}$ \\
\hline$\%$ informes corregidos & $\begin{array}{l}\text { nº exámenes corregidos x } 100 \\
n^{\circ} \text { exámenes recibidos }\end{array}$ & $\leq 0,05 \%$ & $\begin{array}{l}\text { Suma de datos acumulados por cua- } \\
\text { trimestre }\end{array}$ \\
\hline $\begin{array}{l}\text { \% de aviso de valores } \\
\text { de alerta (VA) al médico } \\
\text { tratante antes de } 30 \text { min }\end{array}$ & $\begin{array}{l}\mathrm{n}^{\circ} \text { de VA avisados antes de los } 30 \\
\text { min } \times 100 \\
\mathrm{n}^{\circ} \text { total de VA detectados }\end{array}$ & $\geq 90 \%$ & $\begin{array}{l}\text { Suma de datos acumulados por cua- } \\
\text { trimestre }\end{array}$ \\
\hline
\end{tabular}

${ }^{*}$ CAP: Colegio de Patólogos Americanos, **PEEC, ISP: Programa de Evaluación Externa de la Calidad, Instituto de Salud Pública de Chile.

\section{Fase Analítica}

De los indicadores de esta fase, el indicador de porcentaje de concordancia del Gram del hemocultivo con la identificación final del cultivo fue de 99,5\% (431 láminas de Gram concordantes de 433 láminas totales leídas) y de 99,4\% (472 láminas de Gram concordantes de 475 Gram totales) en el primer y segundo semestre respectivamente, siendo la meta $\geq 98 \%$.

En indicador relativo participaciones en En- cuestas de Control de Calidad Externo, se obtuvo $97,5 \%$ de participaciones correctas (1.749 resultados correctos de 1794 exámenes totales evaluados), superando la meta establecida de 95\%. El SLCPUC participa en 135 encuestas del Colegio de Patólogos Americanos, que controlan 457 exámenes diferentes (de un total de 553 realizados) y todas las áreas de diagnóstico del laboratorio (química clínica, hematología, coagulación, toxicología, microbiología, etc). 
Indicadores de calidad de laboratorio hospitalario - A. M. Guzmán et al

Tabla 3. Indicadores de la fase pre-analítica implementados en Laboratorio de Urgencia del Hospital Clínico UC (área de atención cerrada)

\begin{tabular}{|c|c|c|c|c|c|}
\hline \multirow[t]{2}{*}{ Indicador } & \multirow[t]{2}{*}{ Fórmula } & \multirow[t]{2}{*}{ Meta } & \multicolumn{3}{|c|}{ Resultados } \\
\hline & & & $\begin{array}{c}1 \mathrm{er} \\
\text { cuatrimestre }\end{array}$ & $\begin{array}{c}2^{\circ} \\
\text { cuatrimestre }\end{array}$ & $\begin{array}{c}\mathbf{3 e r}^{\mathrm{er}} \\
\text { cuatrimestre }\end{array}$ \\
\hline $\begin{array}{l}\text { Solicitud } \\
\text { de nueva } \\
\text { muestra }\end{array}$ & $\begin{array}{l}\mathrm{n}^{\circ} \text { de solicitudes } \\
\text { de nueva muestra } \\
\mathrm{X} 100 / \mathrm{n}^{\circ} \text { total de } \\
\text { exámenes recibidos }\end{array}$ & $\leq 0,5 \%$ & $\frac{719 \times 100}{84.651}=0,85$ & $\frac{939 \times 100}{99.977}=0,94$ & $\frac{792 \times 100}{95112}=0,83$ \\
\hline $\begin{array}{l}\text { Cumplimiento } \\
\text { tiempos de } \\
\text { traslado }\end{array}$ & $\begin{array}{l}\mathrm{n}^{\circ} \text { exámenes reci- } \\
\text { bidos en tiempo } \\
\text { adecuado x } 100 / \\
n^{\circ} \text { total de exámenes } \\
\text { recibidos }\end{array}$ & $\geq 80 \%$ & $\frac{24.668 \times 100}{28.390}=87$ & $\frac{26.142 \times 100}{31.521}=83$ & $\frac{24.256 \times 100}{30.059}=81$ \\
\hline $\begin{array}{l}\text { \% de botellas } \\
\text { de hemo- } \\
\text { cultivo con } \\
\text { volumen } \\
\text { adecuado }\end{array}$ & $\begin{array}{l}\mathrm{n}^{\circ} \text { botellas hemo- } \\
\text { cultivo con volumen } \\
\text { adecuado } \times 100 / \\
\mathrm{n}^{\circ} \text { total botellas he- } \\
\text { mocultivo tomadas }\end{array}$ & $\geq 80 \%$ & $\frac{747 \times 100}{2.090}=35,7$ & $\frac{798 \times 100}{2.245}=35,5$ & $\frac{874 \times 100}{2.282}=38,3$ \\
\hline
\end{tabular}

Se incluye la fórmula para una mejor interpretación de los datos. Se destacan con fondo gris aquellos que no cumplen la meta establecida.

Tabla 4. Indicador solicitud de nueva muestra desglosado por servicio clínico (Meta: $\leq \mathbf{0 , 5 \% )}$

\begin{tabular}{|lcccc|}
\hline Servicio & $\begin{array}{c}\text { 1er cuatrimestre } \\
\text { Indicador (\%) }\end{array}$ & $\begin{array}{c}\mathbf{2}^{\mathbf{0}} \text { cuatrimestre } \\
\text { Indicador (\%) }\end{array}$ & $\begin{array}{c}\text { 3er cuatrimestre } \\
\text { Indicador (\%) }\end{array}$ & $\begin{array}{c}\text { Total año } \\
\text { Indicador (\%) }\end{array}$ \\
Anestesia y recuperación & 0,0 & 1,2 & 0,2 & 0,4 \\
Cirugía & 1,3 & 1,6 & 1,3 & 1,3 \\
Cirugía cardiovascular & 1,2 & 1,5 & 1,0 & 1,2 \\
Inter. médico neurológico & 1,6 & 2,2 & 1,8 & 1,8 \\
Medicina & 1,4 & 1,2 & 1,4 & 1,3 \\
Obstetricia y ginecología & 1,1 & 1,2 & 1,5 & 1,2 \\
\hline Pensionado & 1,0 & 1,3 & 1,2 & 1,2 \\
Unidad coronaria & 2,6 & 2,5 & 2,3 & 2,5 \\
UPC adultos & 1,5 & 1,9 & 1,5 & 1,6 \\
Servicio de urgencia & 0,2 & 0,2 & 0,4 & 0,3 \\
Pediatría & 2,0 & 1,7 & 1,7 & 1,8 \\
\hline UPC pediatría & 0,7 & 0,5 & 0,7 & 0,6 \\
UPC neonatología & 1,4 & 1,5 & 0,8 & 1,2 \\
Neonatología & 1,7 & 1,5 & 1,7 & 1,6 \\
\hline
\end{tabular}




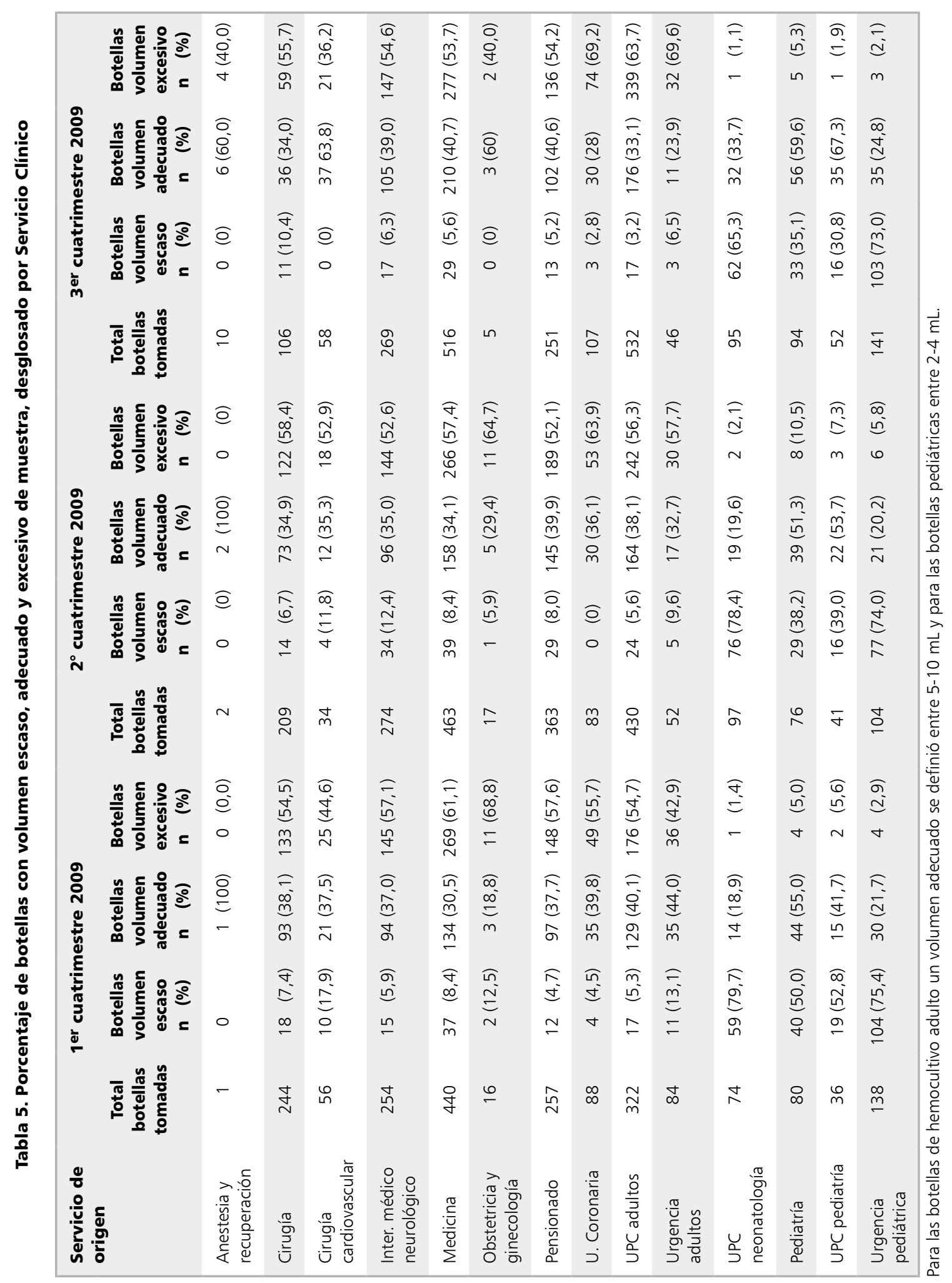




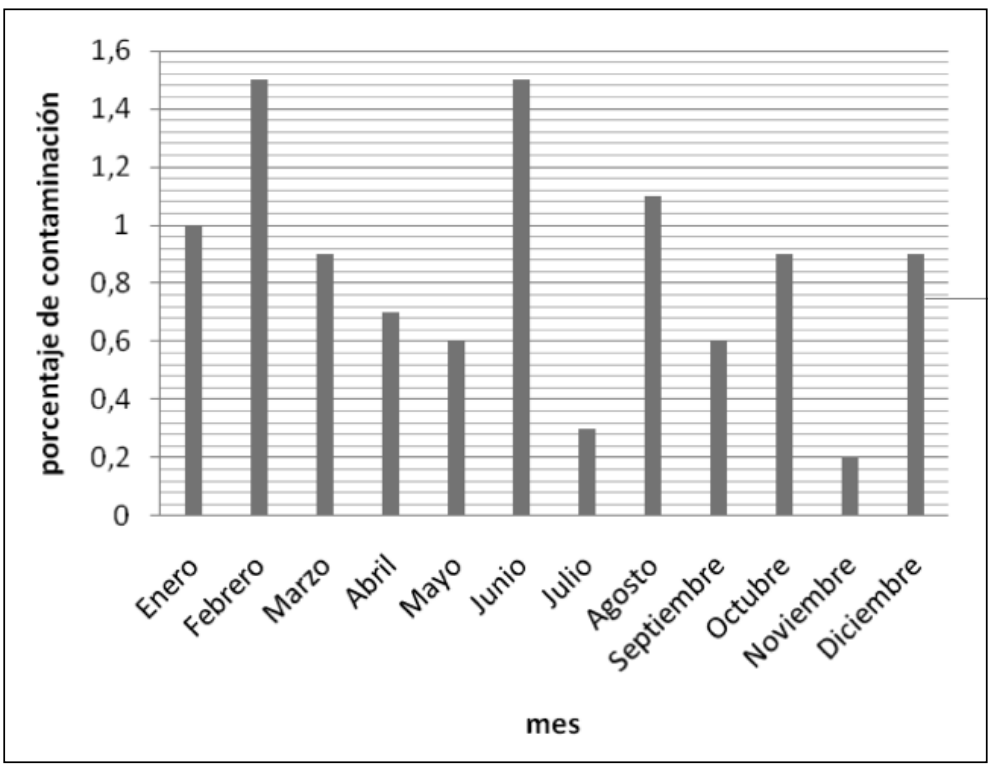

Figura 1. Porcentaje de cumplimiento del indicador de porcentaje de contaminación de los hemocultivos, medido mensualmente (meta $\leq 2 \%$ ).

Tabla 6. Indicadores de la fase post-analítica implementados en Laboratorio de Urgencia del Hospital Clínico UC (área de atención cerrada)

\begin{tabular}{|c|c|c|c|c|c|}
\hline \multirow[t]{2}{*}{ Indicador } & \multirow[t]{2}{*}{ Fórmula } & \multirow[t]{2}{*}{ Meta } & \multicolumn{3}{|c|}{ Resultados } \\
\hline & & & 1er cuatrimestre & $2^{\circ}$ cuatrimestre & 3er cuatrimestre \\
\hline $\begin{array}{l}\text { \% cumplimiento } \\
\text { de plazos de } \\
\text { entrega (pe) de } \\
\text { resultados de } \\
\text { exámenes }\end{array}$ & $\begin{array}{l}\text { no exámenes } \\
\text { dentro plazo } \\
\text { entrega x100/ } \\
\text { n exámenes } \\
\text { recibidos }\end{array}$ & $\geq 80 \%$ & $\frac{1.537 \times 100}{1.614}=95 \%$ & $\frac{1.438 \times 100}{1.604}=90 \%$ & $\frac{1.443 \times 100}{1.610}=90 \%$ \\
\hline $\begin{array}{l}\% \text { informes } \\
\text { corregidos }\end{array}$ & $\begin{array}{l}\mathrm{n}^{\circ} \text { exámenes } \\
\text { corregidos x100/ } \\
\mathrm{n}^{\circ} \text { exámenes } \\
\text { recibidos }\end{array}$ & $\leq 0,05 \%$ & $\frac{21 \times 100}{121.647}=0,02$ & $\frac{21 \times 100}{140.505}=0,01$ & $\frac{16 \times 100}{136.522}=0,01$ \\
\hline $\begin{array}{l}\% \text { de aviso de } \\
\text { valores de alerta } \\
\text { (va) al médico } \\
\text { tratante antes } \\
\text { de } 30 \text { min }\end{array}$ & $\begin{array}{l}\mathrm{n}^{\circ} \text { de VA avisa- } \\
\text { dos antes de los } \\
30 \text { min. X 100/ } \\
n^{\circ} \text { total de valo- } \\
\text { res de alerta }\end{array}$ & $\geq 90 \%$ & $\frac{1.354 \times 100}{1.429}=95 \%$ & $\frac{1.563 \times 100}{1.671}=94 \%$ & $\frac{1.618 \times 100}{1.735}=93 \%$ \\
\hline
\end{tabular}

Se incluye la fórmula para una mejor interpretación de los datos.

\section{Fase Post-analítica}

La Tabla 6 muestra los resultados de los indicadores de esta fase: porcentaje de cumplimiento de plazos de entrega, porcentaje de informes corregidos y porcentaje de aviso de valores de alerta antes de los 30 minutos. En todos, las cifras logradas superan las metas fijadas.

\section{Discusión}

Una forma de mejorar la calidad de la atención en las instituciones de salud es implementar la medición de indicadores de calidad orientados a monitorizar los principales procesos que ocurren en ellas y la posterior implementación 
de acciones de mejora para alcanzar las metas establecidas.

En el área de Medicina de Laboratorio existen algunas publicaciones con datos locales de indicadores, pero aún no existen recomendaciones de consenso de expertos o instituciones que definan indicadores mínimos u obligatorios ni menos aún, metas a lograr. El año 2009, el Clinical Laboratory Standards Institute (CLSI) publicó una propuesta sobre el desarrollo de indicadores de calidad en los laboratorios clínicos, pero no es todavía un documento aprobado y sólo se refiere en forma general al proceso de implementación de indicadores ${ }^{1}$.

Por lo tanto, cada laboratorio debe establecer según su realidad, complejidad, herramientas informáticas disponibles, tipo de pacientes atendidos, etc. qué indicadores son posibles y relevantes de implementar. La relevancia estará definida, por ejemplo, por la importancia que tiene el proceso que se está vigilando en el cuidado del enfermo, porque es un área dónde se han identificado errores en forma frecuente, etc.

De los 9 indicadores implementados en atención cerrada podemos comentar:

\section{Solicitud de nueva muestra}

La proporción de muestras rechazadas varía en diferentes publicaciones entre $0,3 \%$ y $0,8 \%$. Sin embargo, se ha informado que el porcentaje de rechazos para un Servicio de Urgencia es de hasta 2,2\% ${ }^{12-14}$. En nuestro hospital el porcentaje promedio de muestras rechazadas es alto, con algunos servicios que llegan a cifras del $2,5 \%$. Sin duda, constituye un tema a analizar en conjunto con el Servicio de Enfermería. Contrariamente a lo publicado, el porcentaje de rechazos para el Servicio de Urgencia es el más bajo del hospital, lo que señala que podemos globalmente mejorar nuestras cifras.

\section{Cumplimiento de tiempos de traslado}

La supervisión de los tiempos de traslado es fundamental para asegurar que las muestras que entran a análisis conserven estables los analitos que se desean estudiar. El tiempo máximo de traslado de exámenes al laboratorio, definido por el CLSI, es de 2 horas para exámenes habituales y 15 minutos para aquellos urgentes o en los cuáles se desea medir analitos inestables ${ }^{15}$. Publicaciones disponibles presentan cifras muy bajas de daño de muestras durante el traslado y/o tiempos de trans- porte al laboratorio inadecuados $(0,002 \%)$. En nuestra institución el porcentaje de cumplimiento en este indicador es superior a la meta y sólo debe ser reforzado periódicamente en el personal de enfermería y de laboratorio.

Porcentaje de contaminación de los hemocultivos

Internacionalmente se ha recomendado que el porcentaje de contaminación para las botellas de hemocultivos debe ser menor de $2 \%{ }^{16}$. Hemocultivos tomados con mala técnica de antisepsia y que resultan contaminados inducirán a repeticiones del examen, retraso en el diagnóstico del paciente y en ocasiones uso innecesario o inadecuado de antibióticos. En dos estudios multicéntricos que incluyeron 600 y 300 hospitales, la tasa de contaminación varió de 2,5 a 2,9\% respectivamente ${ }^{17,18}$. Nuestro porcentaje de contaminación varío en el año entre 0,2 y $1,5 \%$, lo cual indica que se está usando una buena técnica aséptica.

\section{Porcentaje de botellas de hemocultivos inoculadas con volumen adecuado de sangre}

Este indicador presentó porcentajes de cumplimiento muy bajos (aprox 37\%) en las tres mediciones realizadas en el año. Este parámetro había sido previamente evaluado en nuestra institución y se conocía que era un punto por mejorar (Sánchez T, Guzmán AM, Espinoza C, de la Cerda G, García P. Rendimiento de los hemocultivos según volumen de sangre inoculada. Congreso Chileno de Infectología 2003). En nuestro hospital se detectó mayoritariamente inoculación con volúmenes excesivos de sangre, salvo en las áreas pediátricas. El punto es que se sabe, que tanto volúmenes excesivos como escasos afectan el rendimiento de los hemocultivos ${ }^{19,20}$ por lo que debe ser corregido en conjunto con enfermería, lo más prontamente posible.

\section{Porcentaje de concordancia del Gram del hemocultivo con la identificación final}

Este indicador está dirigido a evitar el inicio de tratamientos empíricos basados en una tinción de Gram informada erróneamente. La competencia del tecnólogo médico que observa la preparación es fundamental en este indicador. En centros que han vigilado el problema por más de 10 años, la discordancia oscila entre 1 y $5 \%{ }^{21-23}$. Los porcentajes de concordancia logrados en nuestro laboratorio son muy altos, considerando que se realizan 
Indicadores de calidad de laboratorio hospitalario - A. M. Guzmán et al

entre 600-700 hemocultivos mensualmente con una positividad del $10 \%$, por lo que las láminas incorrectamente leídas en el total del año no podrían ser más de 12-14 al año.

\section{Porcentaje de participaciones correctas en encuestas de control de calidad}

Según lo publicado, este indicador varía según el área evaluada $(99,9 \%$ en química, 94,5\% en hematología y $93 \%$ en microbiología ${ }^{10}$ y también según la experiencia del laboratorio en la participación en este tipo de encuestas. Nuestro laboratorio participa hace más de 20 años en estas encuestas y el porcentaje global de resultados aceptables es superior al 97\%.

\section{Porcentaje cumplimiento plazos de entrega}

Los plazos de entrega adecuados son un elemento clave tanto en la eficiencia y efectividad de una institución de salud como en la satisfacción por parte del médico tratante respecto al servicio. Entre los estudios publicados respecto a tiempos de respuesta (TAT) en laboratorios de urgencia, destaca uno realizado por el Colegio de Patólogos Americanos, con más de 500 laboratorios participantes y más de 2 millones de exámenes, dónde se encontró que $11 \%$ de estos exámenes tenían TAT superior a 70 minutos $^{24}$. Otro estudio describió que para el potasio y la hemoglobina los TAT medios para el Servicio de Urgencia fueron $60 \mathrm{y}$ 45 minutos respectivamente ${ }^{25}$. Un trabajo sobre troponina determinó que el 90\% de los resultados estaban disponibles en 91 minutos $^{26}$. Nuestro indicador considera todas las prestaciones solicitadas desde el Servicio de Urgencia y los tiempos de respuesta para cada examen fueron determinados por la Subdirección Técnica del Servicio, los cuales varían entre 15 y 60 minutos. El cumplimiento es alto, teniendo en consideración que en nuestro laboratorio, la primera prioridad para el procesamiento la tienen los exámenes provenientes del Servicio de Urgencia.

\section{Porcentaje informes corregidos}

En un estudio de anatomía patológica que incluyó 1,5 millones de biopsias, la proporción de informes corregidos no superó 2 por $1.000 \operatorname{casos}^{10}$. En otro trabajo realizado en un laboratorio de microbiología el porcentaje de informes corregidos en un período de 9 meses fue $0,3 \%{ }^{27}$. En nuestro laboratorio este porcentaje es de 0,01-0,02\% lo que resulta apropiado a las cifras publicadas. Un análisis adicional por realizar, sería establecer el impacto de estas correcciones en el cuidado del enfermo.

\section{Porcentaje de avisos de valores de alerta antes de los 30 minutos}

Los valores de alerta corresponden a resultados que involucran riesgo para la vida del paciente por lo que deben ser avisados lo antes posible al médico tratante ${ }^{28}$. En la literatura existe gran dispersión respecto al tiempo en que éstos se logran avisar ${ }^{29,30}$ lo que está relacionado a si se dispone o no de tecnologías de apoyo para ubicar al profesional. En nuestro hospital se ha fijado un plazo máximo de 30 minutos para ubicar al médico, lo cual cumplimos en más del 90\% de los valores de alerta detectados ${ }^{31}$. Esto debe ser mejorado con la incorporación de sistemas informáticos de laboratorio de clase mundial, capaces de generar estos avisos al médico tratante en forma automática (por pantalla).

\section{Comentario final}

La incorporación de indicadores de calidad para la monitorización de procesos críticos dentro del laboratorio, constituye indudablemente una oportunidad de identificar áreas por mejorar. Es necesario contar con más publicaciones de este tipo, para poder hacer las comparaciones necesarias y llegar a consensos aplicables y útiles para los laboratorios clínicos y particularmente relevantes para el cuidado del paciente. Localmente debemos preocuparnos por disminuir el porcentaje de muestras rechazadas y mejorar la toma de muestra de los hemocultivos respecto al volumen de sangre inoculado, para lo cual se deberá trabajar conjuntamente con el Servicio de Enfermería de nuestro hospital.

\section{Referencias}

1. García R. El concepto de calidad y su aplicación en Medicina. Rev Med Chile 2001; 129: 825-6.

2. Lohr KN, Donaldson MS, Harris-Wehling J. Medicare: a strategy for quality assurance, V: Quality of care in a changing health care environment. QRB Qual Rev Bull 1992; 18: 120-6. 
3. Manual del Estándar General de Acreditación para prestadores institucionales de atención abierta. Superintendencia de Salud. Edición impresa 2009.

4. Manual del Estándar General de Acreditación para prestadores institucionales de atención cerrada. Superintendencia de Salud. Edición impresa 2009.

5. Norma Chilena Oficial NCh 2547 Of. 2003 Laboratorios Clínicos- Requisitos Particulares para la Calidad y Competencia. Instituto Nacional de Normalización.

6. Howanits P. Errors in Laboratory Medicine. Practical lessons to Improve patient safety. Arch Pathol Lab Med 2005; 129: 1252-61.

7. Ricós C, García Victoria M, de la Fuente B. Quality Indicators and specifications for the extra-analytical phases in clinical laboratory management. Clin Chem Lab Med 2004; 42: 578-82.

8. Plebani M., Ceriotti F., Messeri G., Ottomano C., Pansini N., Bonini P. Laboratory network of excellence: enhancing patient safety and service effectiveness. Clin Chem Lab Med 2006; 42: 150-60.

9. Nevalainen D, Berte L, Kraft CH, Leigh E, Picaso L, Morgan T. Evaluating laboratory performance on quality indicators with the six sigma scale. Arch Pathol Lab Med 2000; 124: 516-9.

10. Shahangian S, Snyder S. Laboratory Medicine Quality Indicators. A review of the literature. Am J Clin Pathol 2009; 131: 418- 31.

11. Clinical and Laboratory Standards Institute (CLSI). Development and use of quality indicators for process improvement and monitoring of laboratory quality; Proposed Guideline. CLSI Document GP35 -P (2009).

12. Dale J, Novis D. Outpatient phlebotomy success and reasons for specimen rejection. Arch Pathol Lab Med 2002; 126: 416-9.

13. Stark A, Jones B, Chapman D, Well K, Krajenta R, Meier FA, et al. Clinical laboratory specimen rejection: association with the site of patient care and patient's characteristics. Arch Pathol Lab Med 2007; 131: 588-92.

14. Jones B, Calam R., Howanits P. Chemistry specimen acceptability: a College of American Pathologists Q-probes study of 453 laboratories. Arch Pathol Lab Med 1997; 121: 19-26.

15. Clinical and Laboratory Standards Institute (CLSI). Procedures for the handling and processing of blood specimens; approved guideline. CLSI Document H18-A (2004).

16. Baron E. Cumitech 1C: Blood Cultures IV. ASM Press (2005).

17. Schifman R, Strand C, Meier F, Howanits P. Blood culture contamination: a College of American Pathologists Q-Probes study involving 640 institutions and 497134 specimens from adult patients. Arch Pathol Lab Med 1998; 122: 216-21.

18. Bekeris L, Tworek J, Walsh M, Valenstein P. Trends in blood culture contamination: a College of American Pathologists Q-Tracks study of 356 institutions. Arch Pathol Lab Med 2005; 129: 1222-5.

19. Weinstein MP, Mirrett S, Wilson ML, Reimer LG, Reller LB. Controlled evaluation of 5 versus 10 milliliters of blood cultured in aerobic BacT/Alert blood culture bottles. J Clin Microbiol 1994; 32: 2103-6.

20. Wilson M., Weinstein M. General principles in the laboratory detection of bacteremia and fungemia. Clin Lab Med 1994; 14: 69-82.

21. Rand K, Tillan M. Errors in interpretation of Gram stains from positive blood cultures. Am J Clin Pathol 2006; 126: 686-90.

22. Sogaard M, Norgaard M, Schonheyder HC. First notification of positive blood cultures and high accuracy of the Gram stain report. J Clin Microbiol 2007; 45: 1113-7.

23. Strand C. Positive blood cultures. Can we always trust the Gram stain? Am J Clin Pathol 2006; 126: 671-2.

24. Steindel S, Novis D. Using outlier events to monitor test turnaround time. Arch pathol Lab med 1999; 123: 60714.

25. Steindel S, Jones B, Dale J, Howanits P. Timeliness of automated routine laboratory tests: a College of American Pathologists Q-Porbes study of 653 institutions. Clin Chim Acta 1996; 251: 25-40.

26. Novis DA, Jones BA, Dale J, Walsh M. Biochemical markers of myocardial injury test turnaround time: a College of American Pathologists Q-Probes study of 7020 troponin and 4368 creatine kinase-MB determinations in 159 institutions. Arch Pathol Lab Med 2004; 128: 158-64.

27. Yuan S, Astion M, Schapiro J, Limaye J. Clinical Impact Associated with Corrected Results in Clinical Microbiology Testing. J Clin Microbiol 2005; 43: 2118-93.

28. Lundberg GD. When to panic over abnormal values. MLO Med Lab Observ 1972; 4: 47-54.

29. Howanitz PJ, Steindel SJ, Heard NV. Laboratory critical values policies and procedures: a College of American Pathologists Q-Probes Study in 623 Institutions. Arch Pathol Lab Med 2002; 126: 663-9.

30. Wagar E, Friedber R, Souers R, Stankovic A. Critical values comparison: a College of American Pathologists Q-Probes Survey of 163 Clinical Laboratories. Arch Pathol Lab Med 2007; 131: 1769-75.

31. Guzmán AM, Solari S, Lagos M, Poggi H, Sánchez T, Madrid A, et al. Laboratory alert value reporting by the clinical laboratory at an academic medical network. Rev Med Chile 2009; 137: 1137-44. 